\title{
Compound Archimedean Copulas
}

\author{
Moshe Kelner ${ }^{1}$, Zinoviy Landsman² \& Udi E. Makov² \\ ${ }^{1}$ Department of Statistics, Haifa University, Israel \\ ${ }^{2}$ Actuarial Research Center, Haifa University, Israel \\ Correspondence: Moshe Kelner, Department of Statistics, Haifa University, Israel
}

Received: March 9, 2021 Accepted: April 21, 2021 Online Published: April 24, 2021

doi:10.5539/ijsp.v10n3p126 URL: https://doi.org/10.5539/ijsp.v10n3p126

\begin{abstract}
The copula function is an effective and elegant tool useful for modeling dependence between random variables. Among the many families of this function, one of the most prominent family of copula is the Archimedean family, which has its unique structure and features. Most of the copula functions in this family have only a single dependence parameter which limits the scope of the dependence structure. In this paper we modify the generator of Archimedean copulas in a way which maintains membership in the family while increasing the number of dependence parameters and, consequently, creating new copulas having more flexible dependence structure.
\end{abstract}

Keywords: compound Archimedean copula, dependence structure, association measures, finite tail dependence

\section{Introduction}

Dependence between variables has raised much research interest, where the challenge has always been to find a suitable multivariate distribution with which to model it. One promising direction is based on copula functions which provide a powerful tool geared to building multidimensional distributions with given marginals. By Sklar's Theorem (Sklar's, 1959), every multivariate cumulative distribution function $F\left(X_{1}, \ldots, X_{d}\right)=P\left(X_{1} \leq x_{1}, \ldots, X_{d} \leq x_{d}\right)$ of a random vector $\left(X_{1}, \ldots, X_{d}\right)$ can be uniquely written in term of the separate parts of its marginals $F_{i}\left(x_{i}\right)=P\left(X_{i} \leq x_{i}\right)$ which are set of univariate distributions, and copula $C$, which holds the dependence structure between them, such as

$$
\begin{aligned}
F(\mathbf{x}) & =P\left(X_{1} \leq x_{1}, \ldots, X_{d} \leq x_{d}\right)=C\left(F_{1}\left(x_{1}\right), \ldots, F_{d}\left(x_{d}\right)\right), \\
x_{i} & \in(-\infty, \infty), \quad i=1, \ldots, d .
\end{aligned}
$$

By Nelsen (2006), the copula function must meet three properties. 1. For $u_{i}=F_{i}\left(x_{i}\right)$ when at least one of the marginals has zero value then $C\left(u_{1}, \ldots, 0, \ldots, u_{d}\right)=0,2$. if all marginals except for $u_{i}$ are equal to one, then $C\left(1, \ldots, u_{i}, \ldots, 1\right)=u_{i}$, and 3. $C$ is a d-dimensional non-decreasing function, i.e. $\sum_{i_{1}=1}^{2} \ldots \sum_{i_{d}=1}^{2}(-1)^{i_{1}+\ldots+i_{d}} C\left(u_{1, i_{1}}, \ldots, u_{d, i_{d}}\right) \geq 0$, for any $[a, b] \in(0,1)^{d}$ ordered $a_{i}<b_{i}$ and $u_{j, 1}=a_{j}, u_{j, 2}=b_{j}$ for $j=1, \ldots, d$. This function has been comprehensively researched by Joe (1997), Nelsen (2006), and Durante \& Sempi (2015) to mention only a few. See also references by Druet \& Kotz (2001), and Genest \& MacKay (1986). There are several families of copulas, the most common are the elliptical, which developed from an elliptically distributed random variables, the extreme value copula, which enables a suitable dependence structure for rare events, and the Archimedean family. The advantage of the Archimedean family is the unique structure that is expressed in its generator function. Different choices of generator functions yield different copulas with their particular expression of dependence. Many interesting parametric functions belong to this family, which contains a wealth of dependence structures (Embrechts et al., 2001). Among the most common are the Clayton copula (1978) in which the tails of the distribution are more dependent on the negative tail than on the positive, Frank (1979) which is symmetric Archimedean copula and Gumbel (1960) in which the tails of the distribution are more dependent on the positive tail than on the negative.

Table 1. Examples of families of Archimedean copulas

\begin{tabular}{cccc}
\hline Family & $C_{\theta}(u, v)$ & $\varphi_{\theta}(t)$ & Range of $\theta$ \\
\hline Clayton & {$\left[\max \left(u^{-\theta}+v^{-\theta}-1,0\right)\right]^{-\frac{1}{\theta}}$} & $\frac{1}{\theta}\left(t^{-\theta}-1\right)$ & $\theta \in[-1, \infty) \backslash\{0\}$ \\
Gumble & $\exp \left(-\left[(-\ln u)^{\theta}+(-\ln v)^{\theta}\right]^{-\frac{1}{\theta}}\right)$ & $(-\ln (t))^{\theta}$ & $\theta \in[1, \infty)$ \\
Frank & $-\frac{1}{\theta} \ln \left[1+\frac{\left(e^{-\theta u}-1\right)\left(e^{-\theta v}-1\right)}{e^{-\theta}-1}\right]$ & $-\ln \left(\frac{e^{-\theta t}-1}{e^{-\theta}-1}\right)$ & $\theta \in(-\infty, \infty)$ \\
\hline
\end{tabular}


A two-dimensional Archimedean copula is denoted by:

$$
C_{\theta}(u, v)=\varphi_{\theta}^{-1}\left(\varphi_{\theta}(u)+\varphi_{\theta}(v)\right),
$$

where $\varphi_{\theta}(t)$ is the Archimedean generator and $\theta$ is the dependence parameter. The generator $\varphi$ is a continuous, strictly decreasing convex function $\varphi:[0,1] \rightarrow[0,+\infty]$ such that $\varphi_{\theta}(1)=0$. Kimberling (1974) proved that $\varphi_{\theta}(t)$ is a completely monotone function. Schweizer and Sklar (1983) showed that $\varphi_{\theta}^{-1}(t)$ induces a bivariate copula if it is convex. McNeil $\&$ Neslehova (2009) showed that the necessary and sufficient condition for d-dimensional copula is for $\varphi_{\theta}^{-1}(t)$ to be dmonotone on $[0, \infty)$, i.e., to satisfy $(-1)^{k} \frac{d^{k} \varphi_{\theta}^{-1}(t)}{d t^{k}} \geq 0$ on $[0, \infty)$ and $k \in[1, d-2]$. Many properties of the Archimedean copulas and their generators are introduced and have been proven in Nelsen (2006). Some of them listed as follows:

- The pseudo-inverse of the generator $\varphi$ is the function $\varphi_{\theta}^{[-1]}(t)=\left\{\begin{array}{cc}\varphi_{\theta}^{-1}(t), & 0 \leq t \leq \varphi_{\theta}(0), \\ 0, & \varphi_{\theta}(0) \leq t \leq \infty\end{array}\right.$, Nelsen (2006,4.1.2).

- The distribution function of Archimedean copula $C$ with generator $\varphi_{\theta}(t)$ is denoted by $K_{C}(t)=t-\frac{\varphi_{\theta}(t)}{\varphi_{\theta}^{\prime}\left(t^{+}\right)}$, Nelsen $(2006,4.3 .4)$.

- The density of Archimedean copula $C$ is given by $c_{\theta}(u, v)=-\frac{\varphi^{\prime \prime}(C(u, v)) \varphi^{\prime}(u) \varphi^{\prime}(v)}{\left[\varphi^{\prime}(C(u, v))\right]^{3}}$, Nelsen $(2006,4.3 .6)$.

Archimedean copulas have been used in different fields, such as actuarial science (Albrecher et al., 2011, Thilini et al., 2020), finance risk models (McNeil et al., 2005), portfolio allocations (Hennessy \& Harvey, 2002), and hydrology (Chen and Guo, 2019). Several researchers have been involved in generating new Archimedean copulas, using its generator. Joh and $\mathrm{Hu}$ (1996) introduced families of multivariate copulas with tractable dependence structure, which was obtained by a mixture of a distributions called max-infinitely divisible. Genest et al., (1993) showed five different ways of generating alternative models having an Archimedean generator. The methods were right and left composition, scaling, composition via exponentiation, and linear combination. As an example, they generated a new generator which is a combination of Clayton's Frank's and Gummble's bivariate copulas, given by

$$
\varphi_{\alpha, \beta, \gamma}(t)=\log \left(\frac{1-(1-\gamma)^{\beta}}{1-\left(1-\gamma t^{\alpha}\right)^{\beta}}\right), \quad \alpha>0, \beta>1,0<\gamma<1,0<t \leq 1
$$

Morillas (2005) introduced a method designed to produce new copulas such that $C_{\varphi}\left(x_{1}, \ldots, x_{n}\right)=\varphi^{[-1]}\left(C\left(\varphi\left(x_{1}\right), \ldots, \varphi\left(x_{n}\right)\right)\right)$. She showed sufficient conditions for the new copula but didn't investigate its behavior as compared with that of the original. Spreeuw (2010) presented a flexible family of Archimedean copula where the inverse of an Archimedean generator was generated from $\psi$, a utility function which is nondecreasing and concave. He assumed that $\psi$ defined on $[0,1]$ so $-\psi$ is strictly decreasing and convex and could therefore serve as a generator. He transformed $\psi$ in order to get $\psi(0)=-1$ and defined an Archimedean generator of the form $\varphi(s)=\max [1+\beta(\psi(0)-\psi(s)), 0], s \geq 0, \beta>0$. Bernardino \& Rulliere (2017) proposed conversion of the generator that allowed choosing an upper tail dependence without changes in the shape of the copula. They changed only part of a given generator and called it an Upper-Patched generator because the transformation is local and affects only the upper tail dependence. The new generator is given by $\varphi(t)=P_{d-1}(t)+\left(1-P_{d-1}(t)\right) \varphi_{D}(t)$, where $t<t_{0}, \varphi_{D}(t)$ is a non-strict generator with endpoint $d_{0} \leq t_{0}$ and $u_{2}:=\max \left(u_{1}, u_{2}\right)$. Xie et al., (2017) extended the Durante copula to a multivariate case by applying Marshall-Olkin distribution ideas (Marshall \& Olkin, 1967). In our work we choose a different approach for enriching the Archimedean family and to apply it for two dimensions. We intend to replace $\theta$, the generator parameter by new parameters, and propose a methodology for generating new copulas characterized by enhanced structures and improved properties. In section 2, we introduce a compounding method and the notion of compound generator. A short introduction of dependence measures is given in section 3. An example of generating a compound copula and a comparison of the original and the resulting compound copula is given in section 4 . Conclusions are given in section 5.

\section{Compound Archimedean Copula}

In this paper we present a tool for generating new Archimedean copulas and we provide an extension to this family by creating new generators. This is achieved by using a compound of an existing generator with respect to $g_{\eta}(\theta)$, a probability density of the dependence parameter $\theta$,

$$
\varphi_{M}(t)=\int_{\Theta} \varphi_{\theta}(t) g_{\eta}(\theta) d \theta
$$


where $M$ denotes compound. We now give sufficient conditions on the new generator guaranteeing that the resulting copula belongs to the Archimedean family.

Theorem 1 Let $\varphi_{M}(t)=\int_{\Theta} \varphi_{\theta}(t) g_{\eta}(\theta) d \theta$ be a compound of $\varphi_{\theta}(t)$, a generator of an Archimedean copula, with respect to $g_{\eta}(\theta)$, a density function of $\theta$. Then for any $\varphi_{\theta}(t)$ and density function $g_{\eta}(\theta)$ the compound $\varphi_{M}(t)$ is also an Archimedean copula generator.

Proof. We need to show that the inverse of the Archimedean generator $\varphi_{M}^{-1}(t)$ is strictly decreasing and convex and that the generator satisfying $\varphi_{M}(1)=0$.

Using the fact that for Archimedean generator complies $\varphi_{\theta}(1)=0$ we get

$$
\varphi_{M}(1)=\int_{\Theta} \varphi_{\theta}(1) g_{\eta}(\theta) d \theta=0 .
$$

It is obvious that

$$
\varphi_{\theta}^{-1}\left(\varphi_{\theta}(t)\right)=t
$$

By differentiating both sides of Eq. (6) by t, we get

$$
\begin{gathered}
\varphi_{\theta}^{-1 \prime}\left(\varphi_{\theta}(t)\right) \varphi_{\theta}^{\prime}(t)=1, \\
\varphi_{\theta}^{\prime}(t)=\frac{1}{\varphi_{\theta}^{-1 \prime}\left(\varphi_{\theta}(t)\right)} .
\end{gathered}
$$

Taking into account that $\varphi_{\theta}^{-1}(t)$ is strictly decreasing, we conclude that $\varphi_{\theta}^{-1^{\prime}}(t)<0$ and $\varphi_{\theta}^{-1 \prime}\left(\varphi_{\theta}(t)\right)<0$. Finally, by Eq.(8), we obtain that $\varphi_{\theta}^{\prime}(t)<0$. Similarly we get

$$
\varphi_{M}^{\prime}(t)=\frac{1}{\varphi_{M}^{-1 \prime}\left(\varphi_{M}(t)\right)} .
$$

Let $\varphi_{M}^{\prime}(t)$ be the first derivative by tof the compound generator $\varphi_{M}(t)$ such as

$$
\varphi_{M}^{\prime}(t)=\int_{\Theta} \varphi_{\theta}^{\prime}(t) g_{\eta}(\theta) d \theta
$$

Taking into account that $\varphi_{\theta}^{\prime}(t)<0$ we conclude that $\varphi_{M}^{\prime}(t)<0$ and $\varphi_{M}^{-1^{\prime}}\left(\varphi_{M}(t)\right)<0$ and that leads to $\varphi_{M}^{-1 \prime}(t)<0$. Similarly, we get

$$
\frac{d}{d t} \varphi_{M}^{-1 \prime}(t)=\varphi_{M}^{-1 " \prime}(t)=-\frac{\varphi_{M}^{\prime \prime}\left(\varphi_{M}^{-1}(t)\right)}{\left(\varphi_{M}^{\prime}\left(\varphi_{M}^{-1}(t)\right)\right)^{2}} \varphi_{M}^{-1 \prime}(t)
$$

where $\varphi_{M}^{\prime \prime}(t)$ is the second derivative by t, i.e.

$$
\varphi_{M}^{\prime \prime}(t)=\int_{\Theta} \varphi_{\theta}^{\prime \prime}(t) g_{\eta}(\theta) d \theta
$$

Due to the fact that the generator $\varphi_{\theta}(t)$ is convex (Nelsen, 2006. Theorem 4.1.4), we get that $\varphi_{\theta}^{\prime \prime}(t)>0$ and taking into account (12) we get $\varphi_{M}^{\prime \prime}(t)>0$. We can, therefore, conclude that $\varphi_{M}^{\prime \prime}\left(\varphi_{M}^{-1}(t)\right)>0$. Using $\varphi_{M}^{-1 \prime}(t)<0$, from Eq.(11) the desired result $\varphi_{M}^{-1 "}(t)>0$ is obtained.

corollary 2 For any $\varphi_{\theta}(0)<\infty$, the copula $C_{\theta}(u, v)$ is defined as a non-strict copula (Nelsen, 2006), and $C_{M}(u, v)$, the compound copula, is also non-strict, and the compound generator holds the same end value, $\varphi_{M}(0)$ as that of the original generator, $\varphi_{\theta}(0)$.

Proof. Let $\varphi_{\theta}(t)$ be a non-strict generator, then $\varphi_{\theta}(0)$ is a real number smaller than infinity. Let $\varphi_{M}(t)$ be a compound generator defined in Eq.(4) then

$$
\begin{aligned}
\varphi_{M}(0) & =\int_{\theta} \varphi_{\theta}(0) g_{\eta}(\theta) d \theta \\
& =\varphi_{\theta}(0) \int_{\theta} g_{\eta}(\theta) d \theta=\varphi_{\theta}(0)<\infty
\end{aligned}
$$




\section{Measures of Association and Compound Copula}

The most common approach for characterizing a copula is measuring the strength of dependence which the data hold and its asymptotic properties. In this paper, we focus on two key measures of association, Kendall's tau, which is known as a bivariate concordance and discordance measure, and cross-ratio, which describes local dependence. We also examine the finite tail dependence of the compound copula (Sweeting and Fotiou, 2011).

Kendall's tau. Genest and Rivest (1993) showed that for Archimedean copulas this measure is given by:

$$
\tau_{\theta}=1+4 \int_{0}^{1} \frac{\varphi_{\theta}(t)}{\varphi_{\theta}^{\prime}(t)} d t
$$

Let $\varphi_{\theta}(t)$ be an Archimedean generator resulting in Kendall's tau $\tau_{\theta}$ as introduced in Eq.(14). Let $g_{\eta}(\theta)$ be a compounding distribution of $\theta$ used to create a compound generator shown by (4). By substituting $\varphi_{\theta}(t)$ into $\varphi_{M}(t)$ the Kendall's tau for a compound copula is defined as

$$
\tau_{M}=1+4 \int_{0}^{1}\left[\frac{\int_{\theta} \varphi_{\theta}(t) g_{\eta}(\theta) d \theta}{\int_{\theta} \varphi_{\theta}^{\prime}(t) g_{\eta}(\theta) d \theta}\right] d t .
$$

$\tau_{M}$ can be expressed as an expectation of $\tau_{\theta}$ with respect to a specified distribution $g_{\eta}^{*}$, as seen in the following theorem.

Theorem 3 Let the compounding distribution defined in (4), then $\tau_{M}=E_{g_{\eta}^{*}}\left[\tau_{\theta}\right]$, where $g_{\eta}^{*}=\frac{\varphi_{\theta}^{\prime}(t) g_{\eta}(\theta)}{\int_{\theta} \varphi_{\theta}^{\prime}(t) g_{\eta}(\theta) d \theta}$.

Proof.

$$
\begin{aligned}
E_{g_{\eta}^{*}}\left[\tau_{\theta}\right] & =1+4 \int_{\theta} g_{\eta}^{*}\left[\int_{t=0}^{1} \frac{\varphi_{\theta}(t)}{\varphi_{\theta}^{\prime}(t)} d t\right] d \theta . \\
& =1+4 \int_{t=0}^{1} \int_{\theta} \frac{\varphi_{\theta}(t)}{\varphi_{\theta}^{\prime}(t)} \frac{\varphi_{\theta}^{\prime}(t) g_{\eta}(\theta)}{\int_{\theta} \varphi_{\theta}^{\prime}(t) g_{\eta}(\theta) d \theta} d \theta d t \\
& =1+4 \int_{t=0}^{1}\left[\frac{\int_{\theta} \varphi_{\theta}(t) g_{\eta}(\theta) d \theta}{\int_{\theta} \varphi_{\theta}^{\prime}(t) g_{\eta}(\theta) d \theta}\right] d t=\tau_{M} .
\end{aligned}
$$

Cross-ratio. The cross-ratio function is a commonly used tool to describe local dependence between two correlated variables. It can detect characteristics of association that cannot be captured by any other global dependence measures as Kendall's tau (Abrams et al., 2020). Oakes (1989) defined the measure as:

$$
R_{\theta}(u, v)=\frac{C_{\theta}(u, v) \frac{d^{2}}{d u d v} C_{\theta}(u, v)}{\frac{d}{d v} C_{\theta}(u, v) \frac{d}{d u} C_{\theta}(u, v)} .
$$

Positive or negative local dependence and independence at a location $(u, v)$ are obtained for $R_{\theta}(u, v)>1,0<R_{\theta}(u, v)<1$ and $R_{\theta}(u, v)=1$, respectively. Using basic derivative rules he gave a simplified measure for the Archimedean copula, $R_{\theta}(u, v)=r_{\theta}\left\{C_{\theta}(u, v)\right\}=r_{\theta}(s)=\left.\frac{-s \varphi_{\theta}^{\prime \prime}(s)}{\varphi_{\theta}^{\prime}(s)}\right|_{s=C_{\theta}(u, v)}$. For the compound copula $r_{\theta}$ is replaced by $r_{M}$ and $C_{\theta}(u, v)$ is replaced by $C_{M}(u, v)$.

Finite tail dependence. The coefficient of tail dependence measures the amount of dependence in the upper and the lower tail of distribution at the limit. For a copula function this boundary does not always exist. An alternative which we adopt here is to calculate the measure at a finite value $k$, (Sweeting and Fotiou, 2011). The finite upper tail dependence (FUTD) for the compound copula at $k$ is defined as

$$
\lambda_{U}^{(k)}=\frac{1-2 k+C_{M}(k, k)}{1-k},
$$

and the finite lower tail dependence (FLTD) as

$$
\lambda_{L}^{(k)}=\frac{C_{M}(k, k)}{k} .
$$

\section{Generating a Compound Copula: An Example}

In this section, we introduce an example of generating a compound copula. We show the benefits gained by using the compound copula, as compared to the standard Archimedean copula, by comparing the values of the dependence measures discussed above. 


\section{Example 1.}

Let $C_{\theta}(u, v)$ be a copula introduced by Nelsen $(2006,4.2 .8)$

$$
C_{\theta}(u, v)=\max \left[\frac{\theta^{2} u v-(1-u)(1-v)}{\theta^{2}-(\theta-1)^{2}(1-u)(1-v)}, 0\right] .
$$

This copula is an Archimedean copula with generator function

$$
\varphi_{\theta}(t)=\frac{1-t}{1+(\theta-1) t}, \theta>1 .
$$

Note that this a non-strict copula with $\varphi_{\theta}(0)=1$.

Let us assume that $\theta$, the dependence parameter, is distributed

$$
g_{\eta}(\theta)=\frac{1}{\ln (b)-\ln (a)} \frac{1}{\theta}, a<\theta<b .
$$

Then using Eq.(4) we get a compound generator of the form

$$
\begin{aligned}
\varphi_{M}(t) & =\frac{1}{\ln (b)-\ln (a)} \int_{a}^{b} \frac{1}{\theta} \frac{1-t}{1+(\theta-1) t} d \theta \\
& =\frac{1}{\ln (b)-\ln (a)}(\ln (\theta)-\ln ((\theta-1) t+1))_{a}^{b} \\
& =\frac{1}{\ln (b)-\ln (a)}\left(\begin{array}{c}
\log (b)-\ln ((b-1) t+1) \\
-\log (a)+\ln ((a-1) t+1)
\end{array}\right) \\
& =1+\frac{\ln \left(\frac{((a-1) t+1)}{((b-1) t+1)}\right)}{\ln \left(\frac{b}{a}\right)}, \quad \forall b>a,
\end{aligned}
$$

which provides $\varphi_{M}(1)=1+\frac{\ln \left(\frac{a}{b}\right)}{\ln \left(\frac{b}{a}\right)}=1-1=0$, and $\varphi_{M}(0)=1+\frac{\ln (1)}{\ln \left(\frac{b}{a}\right)}=1$, with an inverse

$$
\varphi_{M}^{-1}(t)=\frac{b a^{t}-a b^{t}}{b a^{t}-b a^{t+1}+a b^{t+1}-a b^{t}} .
$$

By placing Eq.(25) and Eq.(23) in Eq.(2), a new compound copula is obtained:

$$
C_{M}(u, v)=\max \left[\frac{b a^{k}-a b^{k}}{b a^{k}-b a^{k+1}+a b^{k+1}-a b^{k}}, 0\right],
$$

for $k=\left(\varphi_{M}(u)+\varphi_{M}(v)\right)=\left(1+\frac{\ln \left(\frac{((a-1) u+1)}{(b-1)+1+1)}\right)}{\ln \left(\frac{b}{a}\right)}\right)+\left(1+\frac{\ln \left(\frac{((a-1) v+1)}{(b-1) v+1)}\right)}{\ln \left(\frac{b}{a}\right)}\right)$,

$\forall b>a$.

Kendall's tau: Using Eq.(14), for the original copula we get

$$
\tau_{\theta}=1-\frac{2(\theta+2)}{3 \theta}
$$

and for the compound generator in Eq.(23) we get 


$$
\begin{aligned}
\tau_{M}= & 1+4\left(\begin{array}{c}
2(a-1)(b-1)\left(\ln \left(\frac{b}{a}\right)\right) \\
a-b
\end{array} \quad \int_{t=0}^{1} \frac{(((a-1) t+1)((b-1) t+1))\left(\ln \left(\frac{b}{a}\right)+\ln \left(\frac{((a-1) t+1)}{((b-1) t+1)}\right)\right)}{+3(a+b-2) \ln \left(\frac{b}{a}\right)+b-a}\right) d t \\
& =1+\frac{4}{6(a-b)}\left[\begin{array}{c}
+3(a+b)) \ln \left(\frac{a}{b}\right) \\
+(2(a-1)(b-1)+3(a-1) \\
-\frac{2(b-1)}{(a-1)}+\frac{3(a+b)}{(a-1)}-\frac{3(a+b)}{(b-1)}+\frac{2(a-1)}{(b-1)} \\
+6 \ln \left(\frac{b}{a}\right)+\frac{(3 a-b-2) \ln (a)}{(a-1)^{2}}+\frac{(a+2-3 b) \ln (b)}{(b-1)^{2}} \\
-\frac{6 a}{(a-1)}+\frac{6 b}{(b-1)}
\end{array}\right] .
\end{aligned}
$$

The cross ratio for the original copula,is given by:

$$
r_{\theta}(s)=\frac{-s\left(\frac{2(\theta-1)^{2}(1-s)}{(1+(\theta-1) s)^{3}}+\frac{2(\theta-1)}{(1+(\theta-1) s)^{2}}\right)}{-\frac{\theta}{(1+(\theta-1) t)^{2}}}
$$

where $s=C_{\theta}(u, v)=\max \left[\frac{\theta^{2} u v-(1-u)(1-v)}{\theta^{2}-(\theta-1)^{2}(1-u)(1-v)}, 0\right], \quad r_{\theta}(s) \in[0,2]$.

For the compound copula we get

$$
\begin{aligned}
r_{M}(s) & =\frac{-s\left[\frac{(b-a)(2(a-1)(b-1) s+a+b-2)}{((a-1) s+1)^{2}((b-1) s+1)^{2} \log \left(\frac{b}{a}\right)}\right]}{-\left(\frac{(b-a)}{((a-1) s+1)((b-1) s+1) \log \left(\frac{b}{a}\right)}\right)} \\
& =\frac{s(2(a-1)(b-1) s+a+b-2)}{((a-1) s+1)((b-1) s+1)},
\end{aligned}
$$

where $s=C_{M}(u, v)$.

The upper and lower tail dependence are given by (18)-(19) with (23) substituted in $C_{M}(u, v)$. We now explore graphically the three measures of dependence discussed above with respect to the original and compound copulas. Figures 1,2 and 3 are related to Kendall's tau, Cross-ratio and FLTD, respectively.

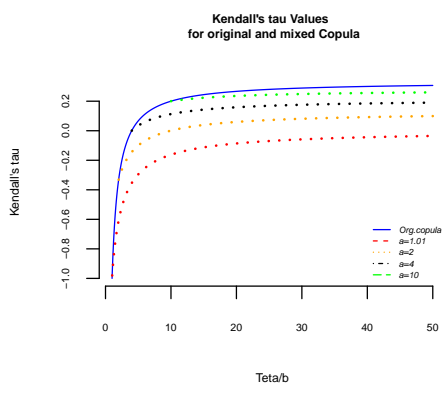

Figure 1. Kendall's tau for mixed and original copula

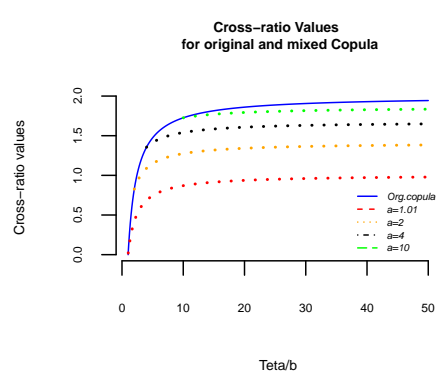

Figure 2. Cross ratio for mixed and original copula

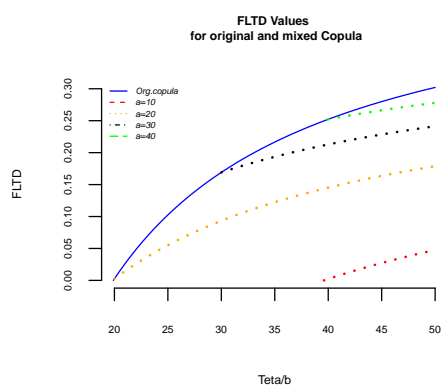

Figure 3. FLTD for mixed and original copula

The blue lines represent the values of the measures as a function of $\theta$ for the original copulas. The other four lines represent the values for the compound copula, where each line corresponds to different fixed values of a and varying values of $b$. From the graphs, it can be seen that the compound copula offers a richer choice of dependence structures.

Figure 4. presents Kendall's tau (x-axis) vs. Cross-ratio values (y-axis) for the original and the compound copulas. The top left represents the original and for the bottom left the compound copula. The right pair is similar to that on the left 
except that Kendall's tau is limited to $(0-0.1)$. We note that while for each value of Kendall's tau there is only one corresponding value of the cross-ratio, the range of such values in the compound copula is much wider. This clearly offers extended modeling possibilities.
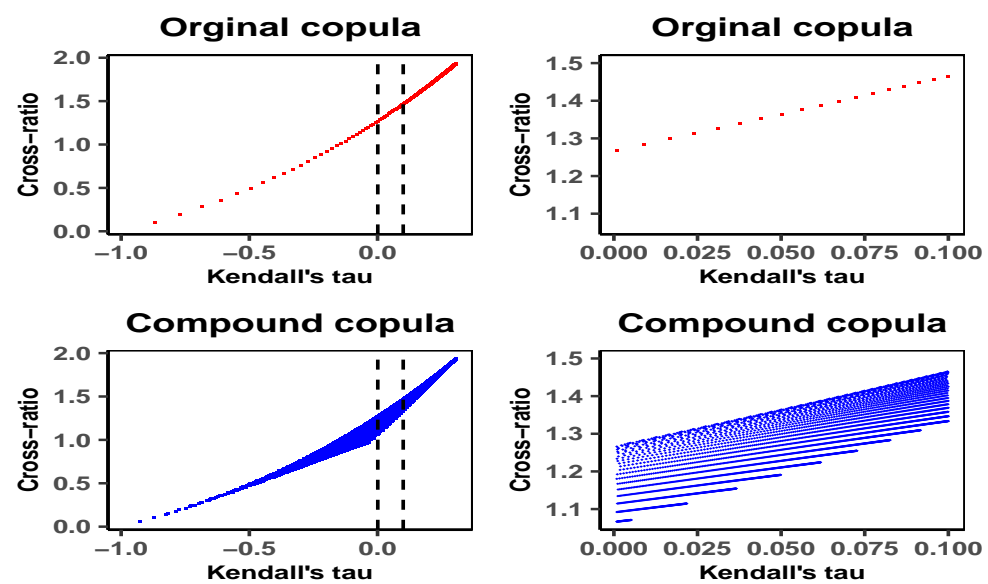

Figure 4. Kendall's tau vs. Cross-ratio for mixed and original copula

\section{Example 2.}

Let $C_{\theta}(u, v)$ be a copula introduced by Nelsen $(2006,4.2 .16)$

$$
C_{\theta}(u, v)=\frac{1}{2}\left[\left(u+v-1-\theta\left(\frac{1}{u}+\frac{1}{v}-1\right)\right)+\sqrt{\left(u+v-1-\theta\left(\frac{1}{u}+\frac{1}{v}-1\right)\right)^{2}+4 \theta}\right] .
$$

With generator function

$$
\varphi_{\theta}(t)=\left(\frac{\theta}{t}+1\right)(1-t), \quad \theta>0
$$

note that this is a strict copula with $\varphi_{\theta}(0)=\infty$. Let us assume that $\theta$, the dependence parameter, is distributed Gamma i.e. $g_{\alpha, \beta}(\theta)=\frac{e^{-\theta \beta} \beta^{\alpha} \alpha^{\alpha-1}}{\Gamma(\alpha)}$. Then using Eq.(4) the compound generator equal to

$$
\begin{aligned}
\varphi_{M}(t) & =\int_{\theta=0}^{\infty} g_{\alpha, \beta}(\theta) \varphi_{\theta}(t) d \theta \\
& =\int_{\theta=0}^{\infty} \frac{e^{-\theta \beta} \beta^{\alpha} \theta^{\alpha-1}}{\Gamma(\alpha)}\left(\left(\frac{\theta}{t}+1\right)(1-t)\right) d \theta \\
& =\frac{(\alpha+\beta t)(1-t)}{\beta t}
\end{aligned}
$$

with an inverse

$$
\varphi_{M}^{[-1]}=\frac{1}{2 \beta}\left((-\alpha-\beta t+\beta)+\sqrt{(\alpha \beta-\beta-t)^{2}-4 \beta t}\right) .
$$

And using Eq.(2) a new compound copula is obtained

$$
\begin{aligned}
C_{M}(u, v)= & \frac{1}{2 \beta}\left(\left(-\alpha-\beta \cdot\left(\left(\frac{(\alpha+\beta u)(1-u)}{\beta u}\right)+\left(\frac{(\alpha+\beta v)(1-v)}{\beta v}\right)\right)+\beta\right)\right)+ \\
& \frac{1}{2 \beta}\left(\sqrt{\left.\begin{array}{c}
\left(\alpha+\beta \cdot\left(\left(\frac{(\alpha+\beta u)(1-u)}{\beta u}\right)+\left(\frac{(\alpha+\beta v)(1-v)}{\beta v}\right)\right)-\beta\right)^{2}+ \\
4 \beta\left(\left(\frac{(\alpha+\beta u)(1-u)}{\beta u}\right)+\left(\frac{(\alpha+\beta v)(1-v)}{\beta v}\right)\right)
\end{array}\right)}\right.
\end{aligned}
$$

which it is a strict copula with $\varphi_{M}(0)=\infty$. We now explore this copula function using two dependence measures. Kendall's tau and Blomqvit's $\beta$, which describe the position of pairs of observations relative to their quadrants and obtained 
by

$$
\beta_{l}=4 \cdot C\left(\frac{1}{2}, \frac{1}{2}\right)-1
$$

For this purpose we substitute (33) and (32) into Kendall's tau Eq.(14), and calculate Blomqvit's $\beta$ by substituting (31) and (35) into Eq.(36) for $u=\frac{1}{2}$ and $v=\frac{1}{2}$. We will explore this two measures numerically. Figure 5 relates to Kendall's tau and Blomqvit's $\beta$.

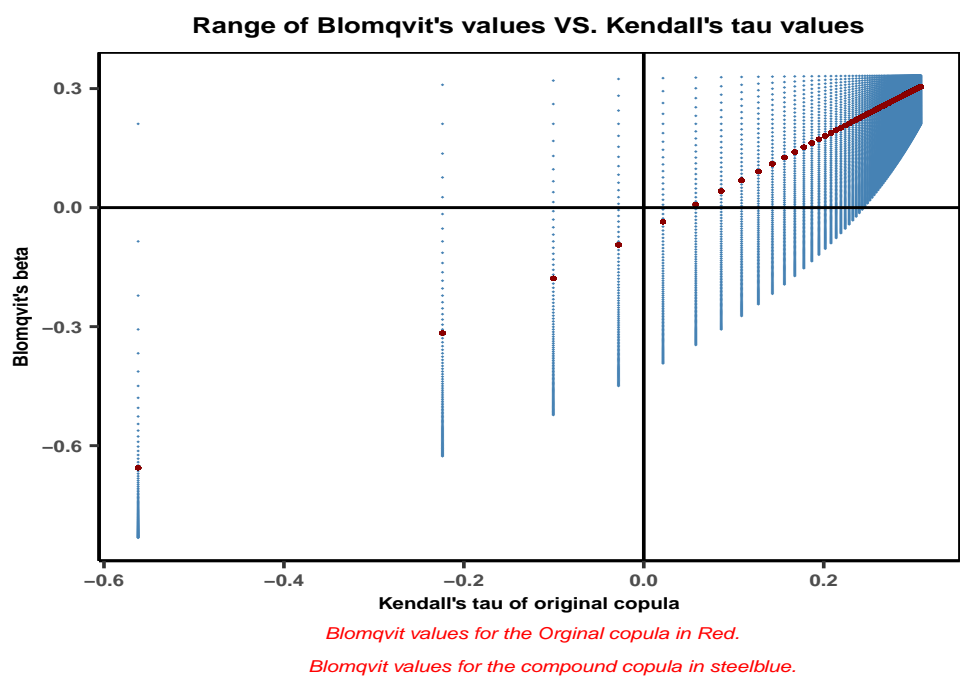

Figure 5. Kendall's tau vs. Blomqvit's $\beta$ for the original and the compound copulas

The red dots represent the values of Blomqvit's $\beta$ corresponding to Kendall's tau in the original copula. We note that for each value of $\tau_{\theta}$ there is a single corresponding Blomqvit's $\beta$. The blue lines represent the values of Blomqvit's $\beta$ corresponding to Kendall's tau in the compound copula. For each $\tau_{\theta}$ there are multiple values of Blomqvit's $\beta$. While for the original copula, positive values of $\tau_{\theta}$ resulted in only positive values of Blomqvit's $\beta$ and negative values of $\tau_{\theta}$ resulted in only negative values of Blomqvit's $\beta$, this restriction is removed when it comes to the compound copula, e.g. in the second quadrant there are positive values of Blomqvit's $\beta$ corresponding to negative values of $\tau_{\theta}$.

\section{Conclusions}

In this paper, we introduce a novel method for generating new members of Archimedean copulas. We use a compound distribution approach by which we compound the generator function of a copula with a density function of its dependence parameter. We therefore create a new compound generator function which subsequently generates a compound Archimedean copula. We demonstrate this process with particular Archimedean copulas and show that the compound copulas offer a higher degree of flexibility in terms of dependence measures.

\section{References}

Abrams, S., Janssen, P., Swanepoel, J., \& Veraverbeke, N. (2020). Non-parametric estimation of the cross-ratio function. Annals of the Institute of Statistical Mathematics, 72, 771-801. https://doi.org/10.1007/s10463-019-00709-3

Albrecher, H., Constantinescu, C., \& Loisel, S. (2011). Explicit ruin formulas for models with dependence among risks. Insurance Math. Econom., 48, 265-270. https://doi.org/10.1016/j.insmatheco.2010.11.007

Bernardino, E. D., \& Rullire, D. (2017). A note on upper-patched generators for Archimedean copulas. Probability and statistics. 21,183-200. https://doi.org/10.1051/ps/2017003

Chen, L., \& Guo, S. (2019). Copulas and Its Application in Hydrology and Water Resources. Springer, New York.

Clayton, D. G. (1978). A Model for Association in Bivariate Life Tables and Its Application in Epidemiological Studies of Familial Tendency in Chronic Disease Incidence. Biometrika., 65, 141C151. https://doi.org/ 10.1093/biomet/65.1.141

Drouet, M., \& Kotz, S. (2001).Correlation and dependence. Imperial College Press, London.

Durante, F., Quesada-Molina, J. J., \& Sempi, C. (2007a). A generalization of the Archimedean class of bivariate copulas. Ann. Inst. Statist. Math., 59, 487-498. https://doi.org/ 10.1007/s10463-006-0061-9

Durante, F., \& Sempi, C. (2015). Principles of copula theory. Chapman and Hall, New York. 
Embrechts, P., Lindskog, F., \& McNeil, A. (2001). Modelling Dependence with Copulas and Applications to Risk Management. Handbook of Heavy Tailed Distributions in Finance, ed. S. Rachev, Elsevier. 8, 329-384.

Frank, M. J. (1979). On the Simultaneous Associativity of $\mathrm{F}(\mathrm{x}, \mathrm{y})$ and $\mathrm{x}+\mathrm{y}-\mathrm{F}(\mathrm{x}, \mathrm{y})$. Aequationes Math. 19, $194 \mathrm{C} 226$. https://doi.org/10.1007/BF02189866

Genest, C., \& MacKay, R. J. (1986b). The joy of copulas: Bivariate distributions with uniform marginal. Amer. Statist. 40, 280-283. https://doi.org/10.2307/2684602

Genest, C., \& Rivest, L. P. (1993). Statistical Inference Procedures for Bivariate Archimedean Copulas. Journal of the American Statistical Association, 88, 1034-1043. https://doi.org/10.2307/2290796

Gumbel, E. J. (1960). Distributions des valeurs extrimes en plusieurs dimensions, Publ. Inst. Statist. Univ. Paris, 9, 171-173.

Hennessy, D., \& Harvey, L. (2002). The use of Archimedean copula to model portfolio allocations. Matematical Finance, 12, 143-154. https://doi.org/10.1111/1467-9965.00136

Joe, H. (1997). Multivariate models and dependence concepts. Chapman and Hall, New York.

Joh, H., \& Hu, T. (1996). Multivariate Distributions from Mixtures of Max-Infinitely Divisible Distributions, Journal of Multivariate Analysis, 57, 240-265. https://doi.org/10.1006/jmva.1996.0032

Kimberling, C. H. (1974). A probabilistic interpretation of complete monotonicity. Equations Math., 10, 152-164. https://doi.org/10.1007/BF01832852

Marshall, A. W., \& Olkin, I. (1967). A multivariate exponential distribution. Amer. Statist. Assoc., 62, 30-44. https://doi/abs/10.1080/01621459.1967.10482885

McNeil, A. J., Rdiger F., \& Embrechts, P. (2005). Quantitative Risk Management: Concepts, Techniques and Tools. Revised edition. Economics Books, Princeton University Press, edition 2.

McNeil, A. J., \& Nešlehová, J. (2009). Multivariate Archimedean copulas, d-monotone functions and L1-norm symmetric distributions. The Annals of Statistics., 37(5B), 3059-3097. https://doi.org/10.1214/07-AOS556

Morillas, M. P. (2005). A method to obtain new copulas from a given one. Metrika: International Journal for Theoretical and Applied Statistics. 61, 169-184. https://doi.org/10.1007/s001840400330

Nelsen, R. B. (2006). An Introduction to Copulas. Second ed. Springer, New York.

Oakes, D. (1989). Bivariate Survival Models Induced by Frailties. Journal of the American Statistical Association, 84, 487-93. https://doi.org/10.2307/2289934

Schweizer, B., \& Sklar, A. (1983). Probabilistic Metric Spaces. North-Holland, New York.

Sklar, A. (1959). Fonctions de rpartition n dimensions et leurs marges. Publications de l'Institut de Statistique de l'Universit de Paris, 8, 229-231.

Spreeuw, J. (2010). Relationships between Archimedean copulas and Morgenstern utility functions. Copula Theory and its Applications, 17, 309-320. https://doi.org/ 10.1007/978 - 3 - 642 - $12465-5 \_17$

Sweeting, P. J., \& Fotiou, F. (2011). Calculating and communicating tail association and the risk of extreme loss A discussion paper. Presented to The Institute and Faculty of Actuaries, London.

Thilini, D. K., Jackie, L., \& David, P. (2020). On the use of Archimedean copulas for insurance modelling. Annals of Actuarial Science, 1-25. https://doi.org/ 10.1017/S1748499520000147

Xie, J., Lin, F., \& Yang, J. (2017). On a generalization of Archimedean copula family. Statistics \& Probability Letters, 125, 121-129. https://doi.org/10.1007/s00500-005-0523-7

\section{Copyrights}

Copyright for this article is retained by the author(s), with first publication rights granted to the journal.

This is an open-access article distributed under the terms and conditions of the Creative Commons Attribution license (http://creativecommons.org/licenses/by/4.0/). 